\title{
ARTIFICIAL INTELLIGENCE METHODS IN DIAGNOSTICS OF ANALOG SYSTEMS
}

\author{
PIOTR BILSKI ${ }^{* * *}$, JACEK WOJCIECHOWSKI * \\ * Institute of Radioelectronics \\ Warsaw University of Technology, ul. Nowowiejska 15/19, 00-665 Warsaw, Poland \\ e-mail: \{pbilski, jwojc\} @ire.pw.edu.pl \\ ${ }^{* *}$ Department of Applied Informatics \\ Warsaw University of Life Sciences, ul. Nowoursynowska 159, 02-776 Warsaw, Poland \\ e-mail:piotr_bilskiesggw.pl
}

\begin{abstract}
The paper presents the state of the art and advancement of artificial intelligence methods in analog systems diagnostics. Firstly, the diagnostic domain is introduced and its problems explained. Then, computational intelligence approaches usable for fault detection and identification are reviewed. Particular groups of methods are presented in detail, explaining their usefulness and drawbacks. Examples, such as the induction motor or the electronic filter, are provided to show the applicability of the presented approaches for monitoring the state of analog objects from engineering domains. The discussion section reviews the presented approaches, their future prospects and problems to be solved.
\end{abstract}

Keywords: fault detection, artificial intelligence, analog systems.

\section{Introduction}

Diagnostics of analog systems is an important issue in technical sciences. The progress of technology allows introducing autonomous systems to everyday life, industrial processes and machinery. Their malfunction could lead to serious threats to human safety. From the economical point of view, the faster the damage is detected and eliminated, the lesser costs of repairs.

Technical diagnostics has two applications. The first one is manufacturing-oriented, aimed at detecting problems with an object at the production stage (such as a proper template for the mask in semiconductor production). The second application area of diagnostics is post-production maintenance, where an object is monitored on-line or periodically to determine its state. This is important for expensive and complex systems. The task is not only to determine incorrect behavior, but also to identify its source. Although multiple approaches have been applied in recent years, the increasing importance of computational intelligence methods has been reported because of their proved high efficiency. Multiple algorithms are known and ready to be used thanks to numerous tools.

The paper is an extension of our previous work (Bilski and Wojciechowski, 2012) and presents an overview of modern Artificial Intelligence (AI) methods applicable to the diagnostics of analog systems. Advantages and characteristic features of particular approaches are discussed. Some taxonomy of modern algorithms and examples of applications are provided. Diagnostic complexity varies for different classes of objects. Therefore, it is not possible to propose universal a method for all tasks. Therefore, the authors focus on linear, lumped and stationary objects.

The structure of the paper is as follows. In Section 2 some fundamentals of technical diagnostics are provided. Section 3 contains examples of analog systems, used to present operation of the methods. Section 4 delivers an overview of artificial intelligence in diagnostics, including data set description. In Section 5 subsequent groups of algorithms are presented from the diagnostic perspective. In Section 6 experimental results are discussed. Section 7 contains an assessment of the reviewed AI methods and discusses future prospects. 


\section{Principles and aims of diagnostics}

Diagnostic aims are identical for any analyzed system, although the details are object-dependent. The System Under Test (SUT) must be monitored to determine if it is in the desired operating state (fault detection). The SUT is faulty if its functioning does not conform to design specification due to changes in its parameters. It is possible for multiple parameters to be out of tolerances but compensating each other.

In some applications (large scale production), fault detection is the sole aim as damaged SUTs are disposed. Such go-no go tests are performed in electronic circuit production, where a single SUT has a small economic value. In other situations, a post-detecting procedure requires to determine which parameter of the SUT is out of tolerance (fault location). The last task is to find the value of the faulty element (fault identification). Both aims apply to discrete and complex SUTs, which are too expensive for complete replacement.

The diagnostic task requires solving a set of the system input-output equations $f$ with respect to the parameter vector $\boldsymbol{p}$ in the time or frequency domain:

$$
\begin{gathered}
\boldsymbol{y}=\boldsymbol{f}(\boldsymbol{p}, \boldsymbol{x}, t), \\
\boldsymbol{y}=\boldsymbol{f}\left(\boldsymbol{p}, \boldsymbol{x}, \boldsymbol{x}^{\prime}, t\right),
\end{gathered}
$$

where $\boldsymbol{x}$ is the vector of the input signals, $\boldsymbol{x}^{\prime}$ is the vector of the first derivatives of the input signals (for dynamic systems), $\boldsymbol{y}$ is the vector of the output signals, and $t$ is time. We need to determine the value of $\boldsymbol{p}$ based on $\boldsymbol{x}$ and $\boldsymbol{y}$, which requires inverting functions which are possibly not one to-one. SUT analysis is based on input-output characteristics. A scheme of the diagnostic procedure is presented in Fig. 1

The SUT can be analyzed in time, frequency, DC or mixed domains. The selection depends on the particular object and its work mode. Linear dynamic SUTs analyzed in the frequency domain exploit characteristic features of the object, like the $3 \mathrm{~dB}$ band. In the time domain the reaction of the SUT to a selected excitation signal is analyzed, from which maximal values or points of zero crossings are extracted.

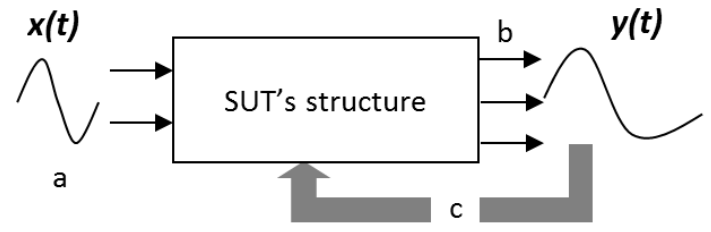

Fig. 1. Schematic of the diagnostics methodology: accessible nodes (a), partially accessible nodes (b), information from the output to determine the state of the SUT (c).
The presented diagnostic tasks are special cases of classification or regression, making them suitable for AI methods. In the first case, the SUT output signal is used to find the category of the fault, based on the set of $n$ actual parameters of $\boldsymbol{p}: \mathbb{R}^{n} \rightarrow Z$. Here $Z$ is a set of integers identifying fault categories. The simplest case is binary classification, which determines whether or not the SUT is faulty. More detailed fault identification and location requires more categories.

Regression enables finding the actual values of SUT parameters. This operation is the mapping of the $n$-dimensional set of characteristic information extracted from the SUT responses $\mathbb{R}^{n}$ into a real number $\mathbb{R}$, i.e., the value of the diagnosed parameter. Each of $n$ parameters must be identified by a separate regression function, which makes the calculations computationally demanding.

The accuracy of diagnostics depends on the selection of nodes at which signals are measured or applied. The most natural is the input node to apply the excitation, and the output node to record responses. Additional nodes are made accessible to create a feasible diagnostic procedure. SUT nodes are divided into three groups:

- accessible-to which one can apply external excitation and measure signals,

- partially accessible-for measurements only,

- not accessible (internal).

The decision which nodes are accessible or partially accessible belongs to the SUT designer. The bigger the number of accessible or partially accessible nodes, the greater the amount of information about the SUT behavior that allows greater accuracy of the diagnostic method. On the other hand, from the perspective of artificial intelligence methods, a too large amount of data may lead to overlearning, decreasing the diagnostic abilities. Therefore, optimal selection of nodes for processing is relevant.

Diagnostic methods are designed to maximize the diagnosability of the SUT, i.e., the ability to detect as many faults as possible. To achieve such a goal, we may play with selection of the set of nodes, the type of excitation signals and analysis domain. Diagnostics is based on the assumption that the SUT structure, nominal values and tolerances of the SUT parameters are known.

The knowledge about the SUT state is extracted from the response signals as the characteristic points (symptoms). Their acquisition requires a thorough understanding of SUT operation to ensure a healthy compromise between the number of stamps and the maximum resolution of the diagnostics. Automated, numerical procedures are applied to extract stamps from SUT responses.

Expert knowledge is required to bind the information in the response with the state of the SUT. Since the 
introduction of AI, this knowledge has been implemented in computer programs, working as a support for the human operator. From this perspective, diagnostics is applied to multiple groups of technical SUTs, such as the following:

- Analog circuits: currently their popularity and usefulness has decreased, as most contemporary applications are provided by integrated circuits. Their diagnostics aims at fault detection. Fault identification and location can be used only as information for the designer. On the other hand, there are applications (such as radio communication) still using discrete systems. Here repairs of a circuit can be viable.

- Electrical machines: motors and power generators. Their models include electrical and mechanical phenomena, making system monitoring difficult. Also, the relation between the identified model parameter and the element of the machine is not always clear. Human expert knowledge is limited, fostering the construction of multiple mathematical models.

- Automation and control systems: such objects are implemented in distributed installations, where multiple sensors and actuators supervise processes. The former provide the diagnostic information, the latter perform the control task. This involves the Instrument Fault Detection and Isolation (IFDI) scheme (Betta and Pietrosanto, 2000). A wide range of methods are used here: neural networks, fuzzy logic or hidden Markov models.

The reasons to implement computational intelligence methods for diagnostics include the expected higher accuracy and bigger systems feasible for diagnostics. The main problems to solve are as follows:

- The presence of additive noise, which degrades the signal and causes errors in stamp extraction. The denoising procedure has limited accuracy and deforms observed signals. There are multiple approaches to denoising (Zhu et al., 2009). Recently, the most popular has been the wavelet transform.

- Tolerances of elements. If deviations of parameters from nominal values are smaller than tolerances, the SUT is considered to be satisfying the design specification. Tolerances introduce uncertainty to the diagnostic procedure, modify shapes of output signals, degrading the accuracy of stamp extraction.

- Ambiguity groups. Their existence depends on the amount of available information about the system behavior. There are two types of ambiguity groups. In the first one, changes in two or more parameters are visible but seen by the diagnostic method as one fault. Fault detection is possible, but identification or location is not. Multiple approaches have been implemented to deal with the problem (Starzyk et al., 2000). The second type is when changes in the parameters compensate each other, so there is no change in the output responses. This makes fault detection impossible.

- Multiple faults. A majority of diagnostic procedures are focused on detecting single faults. This is the most probable situation, but existence of two or more simultaneous faults at the same time is possible. The success rate in detecting multiple parametric faults is much lower than for single faults. Successful attempts to detect multiple catastrophic faults have been made (Tadeusiewicz et al., 2011).

- Analysis of complex systems. An increase in the complexity of the SUT deteriorates its diagnosability. Firstly, the input-output characteristic is not enough to precisely identify and detect all faults. Additional partially accessible nodes are necessary. Another solution is to apply SUT decomposition (Browning, 2001).

- Intensity of the fault. Although classification produces discrete information, the values of the faulty parameter are continuous. If the value of a parameter changes gradually in time, it is considered parametric. Analysis of parametric faults is not only aimed at determining the SUT state, but also at predicting future changes.

Computational intelligence methods are widely used in the diagnostics because of the following:

- Autonomous reasoning to identify the SUT state, i.e., the ability to mimic behavior of the human expert.

- Automatic knowledge extraction from the training information.

- The ability to work with big training sets, which are difficult to analyze by human experts.

- The ability to make decisions in the presence of noise, missing or erroneous data. Additional methods for denoising or data completion can be used at the preprocessing stage. Also there are approaches ensuring the maximum efficiency in the presence of noise (such as support vector machines).

\section{Diagnosed objects}

This section presents two objects used to demonstrate pros and cons of different approaches. They belong to electronic and electrical domains. 
3.1. Fifth order low-pass filter. Analog filters are still popular in some telecommunications applications. Because of the required steep transition bands, higher order (5th and above) filters are used (Karki, 2002). The model of the presented circuit (Fig. 2) was simulated in the SIMULINK environment. Although not realistic in the case of integrated circuits, all nodes were made partially accessible to maximize the amount of data collected from the SUT. All resistors and capacitors were considered potentially faulty. Their nominal values were $R 1=R 2$ $R 3=R 4=R 5=1 \mathrm{k} \Omega, C 1=16 \mathrm{nF}, C 2=19 \mathrm{nF}$, $C 3=13 \mathrm{nF}, C 4=C 5=51 \mathrm{nF}$. Their tolerances were $10 \%$. The band of the nominal filter was $100 \mathrm{~Hz}$ through $10 \mathrm{kHz}$. The SUT was simulated to obtain information about parametric faults ranging from $10 \%$ to $190 \%$ of the nominal value. The excitation signal was a $9 \mathrm{kHz}$ sinusoid (i.e., close to the $3 \mathrm{~dB}$ frequency of the filter). Resolution of the diagnostic module was set to four fault states (see Section 2) for each element. Generated data sets differed in the numbers of examples to check their influence on the diagnostic efficiency of the methods. Examples covered single faults only. Two pairs of learning/testing sets were prepared, containing 70 and 180 experiments, respectively. Each set contains 54 columns (stamps). The number of fault codes was 45 .

3.2. Induction machine. Asynchronous motors are alternate current machines: in the rotor inside the alternate magnetic field created by the current flowing through the stator is made to rotate. The typical model is described by (Wang et al., 2007)

$$
\begin{aligned}
\frac{\mathrm{d} i_{s d}}{\mathrm{~d} t}= & \frac{\beta}{T_{R}} \varphi_{r d}+\beta n_{p} \omega_{r} \varphi_{r q} \\
& -\gamma i_{s d}+\frac{1}{\sigma L_{s}} u_{s d}, \\
\frac{\mathrm{d} i_{s q}}{\mathrm{~d} t}= & \frac{\beta}{T_{R}} \varphi_{r q}-\beta n_{p} \omega_{r} \varphi_{r d} \\
& -\gamma i_{s q}+\frac{1}{\sigma L_{s}} u_{s q}, \\
\frac{\mathrm{d} \varphi_{r d}}{\mathrm{~d} t}= & -\frac{1}{T_{R}} \varphi_{r d}-n_{p} \omega_{r} \varphi_{r q}+\frac{M}{T_{R}} i_{s} d, \\
\frac{\mathrm{d} \varphi_{r q}}{\mathrm{~d} t}= & -\frac{1}{T_{R}} \varphi_{r q}-n_{p} \omega_{r} \varphi_{r d}+\frac{M}{T_{R}} i_{s} q,
\end{aligned}
$$

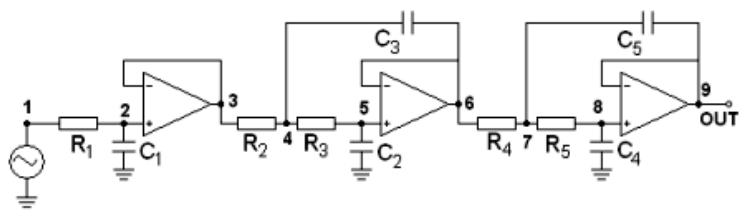

Fig. 2. Structure of the 5th order low-pass filter.

$$
\frac{\mathrm{d} \omega}{\mathrm{d} t}=\frac{M n_{p}}{J L_{r}}\left(i_{s q} \varphi_{r d}-i_{s d} \varphi_{r q}\right)-\frac{C_{e}}{J},
$$

where electrical parameters are $i_{s d}, i_{s q}, u_{s d}, u_{s q}(d$ - and $q$-axis components of stator current/voltage $), \varphi_{r d}, \varphi_{r q}(d-$ and $q$-axis components of rotor flux linkages), $T_{R}$ (the rotor time constant), $n_{p}$ (the number of magnetic pole pairs), $L_{s}, L_{r}, M$ (stator, rotor and mutual inductances). The mechanical parameters are $\omega_{r}$ (the rotor angular speed), $\sigma$ (the total leakage factor), $C_{e}$ (the torque) and $J$ (inertia). The coefficients $\beta$ and $\gamma$ are defined as

$$
\beta=\frac{M}{\sigma L_{s} L_{r}}, \quad \gamma=\frac{R_{s}}{\sigma L_{s}}+\frac{M^{2} R_{r}}{\sigma L_{s} L_{r}} .
$$

The model was simulated in the SIMULINK environment. Seven diagnosed parameters of the motor and their nominal values were $R_{s}=2.25 \Omega, L_{s}=0.1232$ $\mathrm{H}, L_{r}=0.1122 \mathrm{H}, M=0.1118 \mathrm{H}, T_{R}=0.16 \mathrm{~s}, \sigma=$ $0.09, J=0.0504 \mathrm{~kg} \mathrm{~m}^{2}$. The remaining parameters were at their nominal values throughout the test, i.e., $R_{r}=0.7$ $\Omega, n_{p}=3$. The system was simulated to obtain data sets for an ideal model and after considering tolerances of its parameters. The obtained sizes included 28 stamps and, respectively, 49 or 98 examples. Each analyzed parameter was assigned 7 or 14 values, respectively.

Motor responses were analyzed in the startup phase. Four signals were observed: stator current vector $I_{s}$, torque $C_{e}$, angular speed $\omega$, and rotor flux $\varphi_{r}$. For instance, from the torque (Fig. 3), time instants and values of four extremes before reaching the steady state were extracted.

\section{Overview of artificial intelligence methods in diagnostics}

Diagnostic methods have been developed and implemented for the last thirty years. They are divided into the model-based (Simulation After Test, SAT) and data-driven (Simulation Before Test, SBT) ones. In the former, diagnostic information is delivered by the difference between the signal of the model and the actual object. In the latter, the model is simulated beforehand, the knowledge extracted off-line and used during the analysis of measured data. The paper focuses

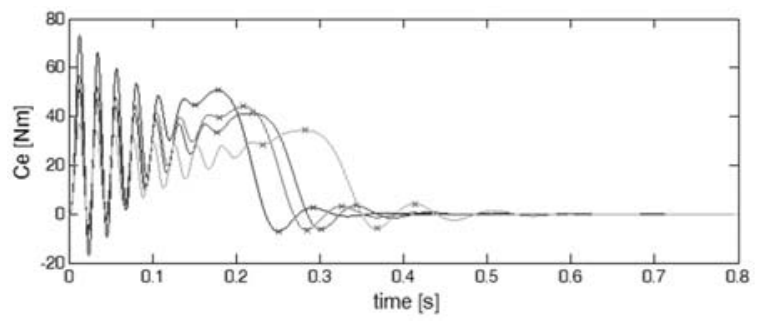

Fig. 3. Induction motor's torque for various values of $R_{s}$. 
on the SBT methodology. Early numerical approaches still remain popular, including sensitivity analysis (Ben Hamida and Kaminska, 1993), mathematical transformations, such as homotopy (Tadeusiewicz and Hałgas, 2007) or multidimensional space analysis (Czaja and Zielonko, 2003). More popular in control domains are diagnostic matrices (Tudoroiu and Zaheeruddin, 2005) and image analysis methods. Dictionary approaches proved to be an effective tool in the diagnostics of analog circuits (Starzyk et al., 2004), with multiple extensions employing also AI methods (Rutkowski and Grzechca, 2001). Some methods require significant computational effort, which twenty years ago was a serious limitation.

Advances in computing methods led to increased interest in heuristic approaches, where the algorithms find dependencies between measured data and the parameter vector $\boldsymbol{p}$ (1). In this section, the taxonomy of AI methods is introduced. Then data sets as the source of knowledge are presented and their specific features for AI-based diagnostics discussed. Finally, selected methods are described and their applications examples presented.

4.1. Taxonomy of AI methods. Although AI is a widely established science with numerous applications, only its fraction useful in diagnostics. Figure 4 presents selected groups of the most useful methods.

The following sections present the most popular and efficient algorithms applied in fault detection. They are grouped according to the method of storing and applying knowledge and diagnostic applications. Artificial Neural Networks (ANNs), rule-based reasoning and statistical approaches are used for classification and regression. Optimization is applied to find the parameters of heuristic methods and testing conditions (test point selection or setting excitation signal frequency) (Rutkowski and

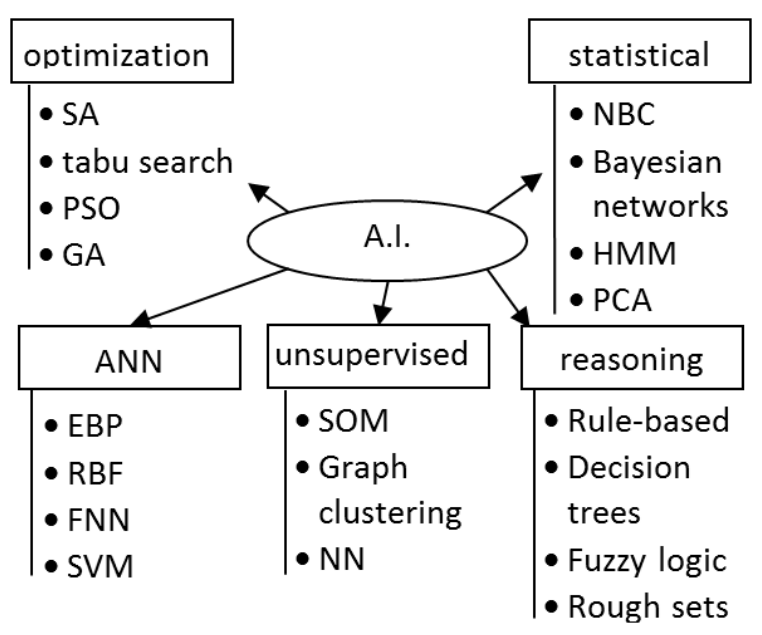

Fig. 4. Classification of AI methods in technical diagnostics.
Zieliński, 2003). Most such problems are NP-hard, requiring heuristic search. Unsupervised learning is used to find dependencies between symptoms disregarding the actual SUT state (Bilski, 2013).

4.2. Measurement data sets. The structure and content of measurement data in the data-driven methods depends on the SUT, but the general form is similar:

$$
D=\left[\begin{array}{cccc}
s_{11} & \cdots & s_{1 m} & c_{1} \\
\vdots & \ddots & \vdots & \vdots \\
s_{n 1} & \cdots & s_{n m} & c_{n}
\end{array}\right]
$$

The table $D$ contains $m$ features (symptoms) $s_{i j}, j=$ $1, \ldots, m$, extracted from observed responses. Rows store stamps of a single faulty model simulation. This allows connecting symptoms with the actual SUT state. The column $c$ contains information about the SUT state. Depending on the diagnostic task (classification or regression), it is either an integer defining the SUT state or a faulty parameter value.

In classification, the range of discrete information depends on the method resolution. In the simplest case, a binary classifier is used. If fault intensity must be identified, additional categories are needed. For example, deviation from the nominal value can be "small", "medium" or "large" (in the positive or negative direction), each identified as a separate category. To isolate a fault-free system, an additional category is needed. Faults are encoded by signed integers (Bilski and Wojciechowski, 2011): “-1" means the value slightly smaller than the nominal, while " 2 " is the value moderately larger than the nominal, etc. This information is combined with the parameter index. As a result, " 0 " is the fault-free state code, " 21 " indicates small positive deviation of the second parameter, while " -32 " is a medium negative deviation of the third parameter.

Simulations are performed after introducing single faults from a fixed category into the SUT. Simulations of the same fault with various intensities lead to registering different symptom values in $D$.

For training and testing an intelligent method, two sets of the form (9) are used: the learning one $L$ to train the diagnostic module, and the testing $T$ one to verify the quality and versatility of the extracted knowledge. An additional validating set $V$ is applied to optimize parameters of the heuristic algorithm. Its purpose is similar to that of $T$. The heuristic learning procedure repeats two stages:

1. Selecting the parameters of the AI method.

2. Training the diagnostic module using the training data set for selected parameters. 
The accuracy of fault detection and identification is measured by the percentage of incorrectly classified examples in $T$ (10). The decision $d_{i}$ made by the diagnostic procedure is compared against the actual SUT state $c_{i} .|D|$ is the number of rows (examples) in the data set,

$$
\boldsymbol{e}=\frac{\left|\left\{d_{i} \neq c_{i}\right\}\right|}{|D|} \times 100 \% .
$$

For the parameter identification task, the regression error is calculated as

$$
\boldsymbol{e}=\frac{1}{|D|} \sum_{i=1}^{|D|}\left(d_{i}-c_{i}\right)^{2} .
$$

The presented methodology belongs to supervised learning, as the expected output is known for each example. This information is used to inform the AI method about the relation between the observed features and the fault source. In the unsupervised learning, examples are unlabeled, so the information $c$ does not exist in the sets, and only symptoms are there. The algorithms find similarities between examples.

During data set generation, the preprocessing stage is optionally implemented. This includes normalization, discretization and minimizing the number of analyzed symptoms. Discretization is required by some algorithms (such as rough sets), where initially continuous values are transformed into numbers of discrete intervals.

\section{Description of diagnostic methods}

This section presents the most popular AI-based approaches to diagnose objects. Their advantage is automation, not requiring interaction with the human operator. Heuristic methods presented in the paper need to be tuned to particular diagnostic case. Algorithms for fast parameter adjustment are developed and tested.

5.1. Logical reasoning methods. These methods come from expert systems, which use easily readable knowledge to detect and identify faults. Rule-based methods exploiting Boolean reasoning were the first to be used for diagnostics. Rules have the form

\section{if premises then fault identification.}

The premises part contains conditions that symptoms must meet to activate the rule. The on-line part of the decision about the SUT state requires determining which rules to activate. Decision trees are an alternative method of representing rules. The leaves represent the fault identification part, while other nodes-the premises part (Fig. [5).

Values at all nodes but leaves contain tests (a symptom identifier and its threshold value). The example travels down the tree starting from the root, comparing the selected symptom value with a threshold at each visited node. Depending on the test result, the example travels to the lower-level node on the left or on the right. When a leaf is reached, the fault identifier it stores is read (Fig. 5 fault codes as in Section 4.2.

Decision trees are more memory efficient than rules. In both cases, algorithms for knowledge extraction (induction tree generation and rules induction, respectively) from the data set were proposed. The knowledge stored by these methods is easily readable and modifiable by the human. The disadvantage is the inability of working in uncertainty conditions (e.g., noise). To activate the rule or redirect the example in the tree, the parameter is confronted against a strict threshold value. This leads to a binary decision of meeting conditions in the premises part ("met" or "not met"). This may degrade the diagnostic accuracy. Another disadvantage of the decision tree is the inability to detect multiple faults, as only single leaves are reached in the reasoning process.

5.2. Some extensions of rule-based approaches. Rule-based systems are supplemented by Fuzzy Logic (FL) and Rough Sets (RSs). Both expand Boolean reasoning, assuming that the analyzed object belongs "to some degree" to the particular category. The premises are not as strict as in the basic rules-based system. The rule may be also activated "to some degree". This facilitates working in the presence of measurement uncertainty.

The FL module (fuzzification, rule activation, aggregation and defuzzification) allows creating a diagnostic system with the number of inputs equal to that of symptoms and the outputs determined by the set of diagnosed parameters. The parallel nature of rules allows multiple fault detection. The fuzziness of input and output variables is represented by membership functions (Fig. 6).

The disadvantage of FL is the absence of a module for extracting knowledge from the data set. It is assumed that the knowledge is delivered by the human expert or the additional algorithm (e.g., decision trees (Bilski and Wojciechowski, 2007)). FL has been successfully used in the diagnostic of electrical machines, servomechanisms

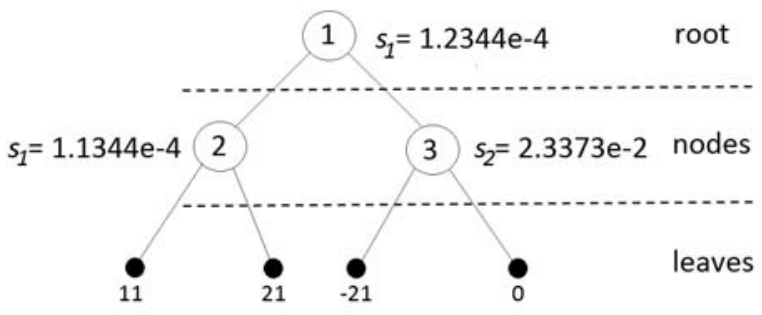

Fig. 5. Example of the decision tree. 
and automotive engines (Simani, 2013).

The idea behind RS is similar to FL. Based on the indiscernibility relation, symptoms are discretized and reduced to generate numerous rules. Discretization transforms continuous values of symptoms into a finite number of intervals, then the reduction is performed to find subsets of symptoms (reducts) sufficient to distinguish between all fault classes. RS cover algorithms for discretization (e.g., Boolean, equal frequency binning, or naive) and reduction (exhaustive, genetic, Johnson, etc.). Based on the information about intervals and reducts produced for them, rules are generated. A problem in RS-based diagnostics is the selection of the best discretization and reduction method. The reasoning procedure is based on the voting mechanism (the fault supported by the greatest number of activated rules is the decision outcome).

The disadvantage of RSs is their inability to detect multiple faults (because of the voting mechanism construction). They also cannot be used for the regression task, either. We have successfully applied them to the diagnostics of an electrical machine and an electronic filter (Bilski and Wojciechowski, 2011). The current research is aimed at finding more accurate and faster discretization and reduction algorithms.

5.3. Artificial neural networks. Alternatively to rule-based methods, artificial neural networks were developed. They are popular in diagnostics (Patan et $a l ., 2008)$ thanks to well-established training algorithms. Initially, Multi-Layered Perceptrons (MLPs) (Fig. 7) were applied to monitor electronic circuits. They are applicable to fault detection or parameter identification. The advantage of ANNs is their versatility, as they can be used to analyze various objects. As the training process involves the main computation effort, many algorithms (such as Levenberg-Marquardt) were proposed. The disadvantage of ANNs is the format of the stored knowledge, difficult to interpret by the human operator. The parallel nature of ANNs made them easy to implement in hardware (such as the digital signal processor (Xue and Jiang, 2006)). MLP parameters (number of layers and neurons in each layer) are set

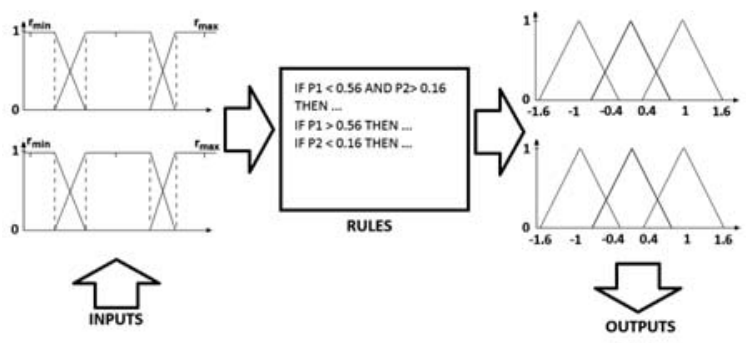

Fig. 6. Fuzzy logic module. for every diagnostic case. MLPs are useful in single or multiple fault detection. In the latter case, coding schemes (such as "One-Vs-All" (OVA) or "Minimum Output Coding" (MOC)) are applied.

ANNs have been applied to identify soft and hard faults in analog circuits (Maiden et al., 1999), monitoring electrical machines (Anand, 2012) and transmission lines. Their modifications are also implemented. RBF ANNs are networks with one hidden layer only, containing neurons with Gaussian activation functions (instead of sigmoidal ones in MLP). Their structure is simpler, but requires more training data to deliver accuracy similar to that of MLP. Fuzzy NNs are also popular, combining rule-based knowledge with training abilities of the network. They have proved to be successful in multiple diagnostic applications (Sałat and Osowski, 2011). Novel training procedures are employed (such as Particle Swarm Optimization (PSO)).

Recently, Support Vector Machines (SVMs) have been proposed, optimally separating objects from different classes (being less susceptible to noise). The design of the SVM diagnostic module requires selecting the optimal kernel function and its parameters. This allows constructing a hyperplane linearly separating different fault classes even in uncertainty conditions (Fig. 8).

If different fault classes cannot be separated in the new feature space, the hyperplane is positioned to minimize the classification (11) or regression (12) error. Automated kernel selection (and its parameters) methods are applied to facilitate the procedure, but frequently RBF turns out to be the best one. Multiple SVMs have to be trained to enable multiple faults classification. Each of them is responsible for one part of the code determining the source of the fault. In the regression

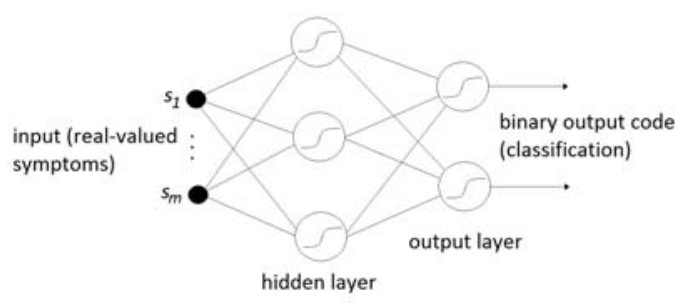

Fig. 7. Perceptron scheme.

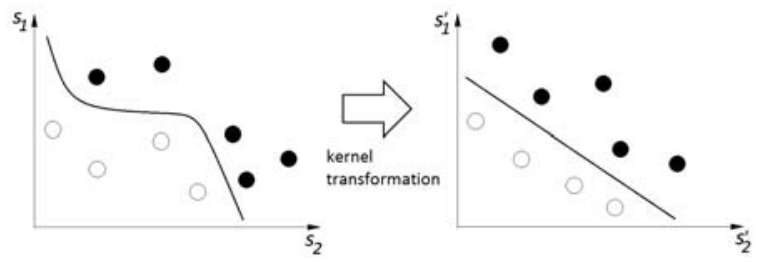

Fig. 8. Idea of transformation between the original and new feature space using the kernel function. 
task, to produce values of each estimated SUT parameter, the same number of SVMs is required. Training SVMs requires the optimization method to adjust the hyperplane (by changing kernel parameters) to fit the data.

SVMs have been used to identify faults in electrical machines (DC and induction motors (Poyhonen et al., 2002)) and electronic circuits (Sałat and Osowski, 2011). Currently new kernels are being developed to provide better diagnostic quality in conjunction with automated design of the network structure.

5.4. Statistical methods. Such methods establish statistical relationships between stamps and fault categories. The decision about the state of the SUT is determined by the number of occurrences of subsequent fault categories in the set and calculating their frequency (probability of occurrence). The Bayes theorem is exploited here, so probabilities depend on the existence of particular symptom values in the example. For each fault category, probabilities are calculated, and the one with the highest value is the diagnostic outcome. The highest computational effort is in data set analysis. The most popular algorithms are the Naive Bayes Classifier (NBC), suitable for discrete values of parameters, and Bayesian networks. If SUT stamps are real-valued, their discretization is required. Statistical approaches detect parametric faults in analog circuits (amplifiers), also using the wavelet transform for preprocessing measured data (Aminian and Aminian, 2001).

Hidden Markov Models (HMMs) are also a popular tool. The HMM is the state machine where various faults are represented by nodes connected by edges (transitions). The latter have probabilistic nature, making it possible to calculate the chances of going from one state to another and selecting the most probable one. The decision about faults is based on the measurements (observable states), leading to the assumption about the hidden states (Fig. 9). HMMs are able to work with both discrete and continuous data. The method is extensively exploited in condition-based maintenance systems, used for monitoring complex and sometimes distributed objects because it allows successful fault prediction. The monitored objects include power plants or production facilities (Smyth, 1994).

Principal Component Analysis (PCA) is used for determining the most important symptoms in the set of examples. Similarly to SVMs, they transform examples from the original domain to the modified one, where they are easier distinguishable. Applications of PCA are twofold: it is used as a standalone diagnostic method (for instance, analysis of sensor arrays (Nowicki et al., 2012)) and as the preprocessing tool before the training stage of the AI approach (often being the preprocessing stage for an ANN).
5.5. Optimization methods. These methods are used in diagnostics for finding the best parameters of heuristic algorithms and simulation or testing conditions (such as the shape of excitation) maximizing diagnostic accuracy. All approaches from Fig. 4 are capable of avoiding local optima. Tuning them requires the training and validating set. The algorithm is trained for the vector of fixed parameters $\alpha$ and evaluated using the evaluation set (Fig. 10). After obtaining the error $\boldsymbol{e}$, the procedure is repeated for the next vector $\alpha$. The lowest error and the corresponding vector $\alpha$ are stored. In continuous problems, Simulated Annealing (SA), PSO and the evolutionary algorithm are usable. In discrete problems (such as optimal test point selection), Tabu Search (TS) is better suited, although the ant colony method should also be considered.

SA processes two $\alpha$ sets at a time: the actual $\alpha_{a}$ and the new one $\alpha_{n}$ (selected randomly from the neighborhood of $\alpha_{a}$ ). Keeping one of them depends on their quality and the probability of selecting the worse solution. The method has been used to optimize parameters of the SVM classifier as well as and regression machines for analog filters and induction machine diagnostics. Its efficiency is comparable to that of genetic algorithms or particle swarm optimization with a shorter time of operation.

PSO uses multiple agents working together to find

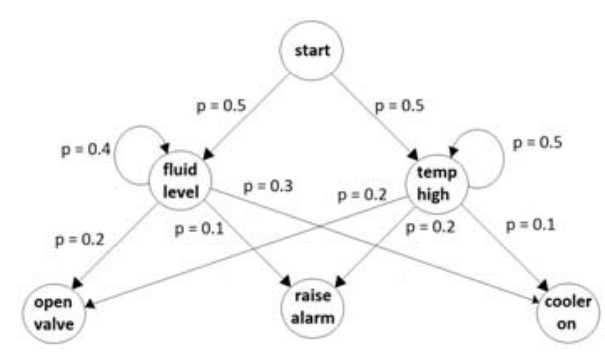

Fig. 9. Hidden Markov model scheme

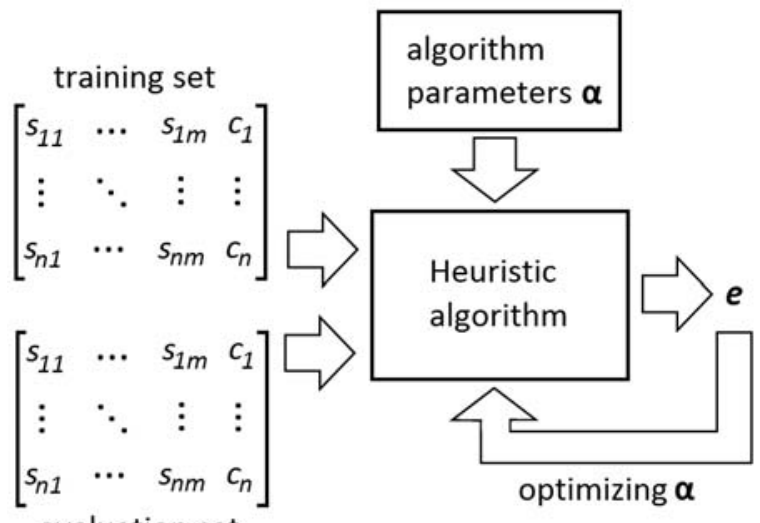

evaluation set

Fig. 10. Heuristic method optimization scheme. 
the best vector $\alpha_{a}$, based on the position and "velocity" of each vector. The method has been used to optimize MLP, RBF and SVM parameters in the diagnostics of electrical machines (Samanta and Nataraj, 2009) or hydraulic systems. Its computational efficiency is higher than in SA, with lower chances of ending in a local optimum.

In TS, two sets of parameters are processed. They are generated from the neighborhood of $\alpha_{a}$ by modifying it to obtain $\alpha_{n}$. The best of them is selected as the new $\alpha_{a}$, provided that it is not on the tabu list (containing the last $n$ solutions, where $n$ is the size of the list). Its content presents forbidden operations for the process of generating new solutions. The tabu list prevents generating the same solutions repeatedly. TS has been successfully used to optimize data for training the neuro-fuzzy inference system, to select the optimal set of nodes in complex SUT diagnostics, and to detect multiple faults in distribution networks.

The EA processes multiple sets of parameters simultaneously, which makes it the most computationally demanding (similarly to PSO). Genetic operations (mutation and crossover) allow obtaining new population $\alpha_{n}$ from the actual one $\alpha_{a}$. This makes the EA insensitive to ending in the local optimum. The method was used to select the parameters of the excitation signal for the SUT (Rutkowski and Zieliński, 2003).

5.6. Unsupervised learning methods. Unsupervised learning is used in diagnostics to find relations in data sets disregarding labels of the simulated examples. In this way, the $D$ set has the form

$$
D=\left[\begin{array}{ccc}
s_{11} & \cdots & s_{1 m} \\
\vdots & \ddots & \vdots \\
s_{n 1} & \cdots & s_{n m}
\end{array}\right]
$$

The aim is to find similar examples based on their symptoms. Thus, it is known which simulations produce similar output. The main concern is similarity, based on the distance between examples. Its values are compared with a threshold. Values below it indicate similar examples, forming a cluster (group). In this way, it is known which examples are similar to each other and what stamp values are most characteristic for the subsequent clusters. There are multiple algorithms for representing relations between unlabeled examples with usually low computational complexity.

The most popular is the Self-Organizing Map (SOM), a one-layered ANN with neurons located on a two-dimensional plane (Fig. 11). Each neuron is potentially responsible for one category. The network shares the same inputs. Their number is determined by symptoms. After presenting an example to the network, all neurons perform computations. The one with the weights closest to the stamp vector (in terms of the selected distance) is the winner. Depending on the training mode, only the winner is trained to respond stronger to this example (Winner Takes All (WTA)), or also a set of its neighbors (Winner Takes Most (WTM)). After presenting the whole set $D$, subsequent neurons are specialized to react strongly to a particular subset of examples. The method was successfully used to monitor power plants and vehicular systems (Svensson et al., 2008), and to adjust the RBF network in induction machine diagnostics (Wu and Chow, 2004).

Alternatively, the graph clustering approach is used for similar aims as with SOMs. Every example from $D$ is a node of a weighted undirected graph. The similarity of nodes is the distance between them $d$ (e.g., the Euclidean distance). Symptoms are often scaled to the same range. Similarity above a predefined threshold determines examples belonging to the same cluster, and other edges are eliminated. In this way, groups of examples are nodes connected to each other (Fig. 12).

Unsupervised learning methods were used to

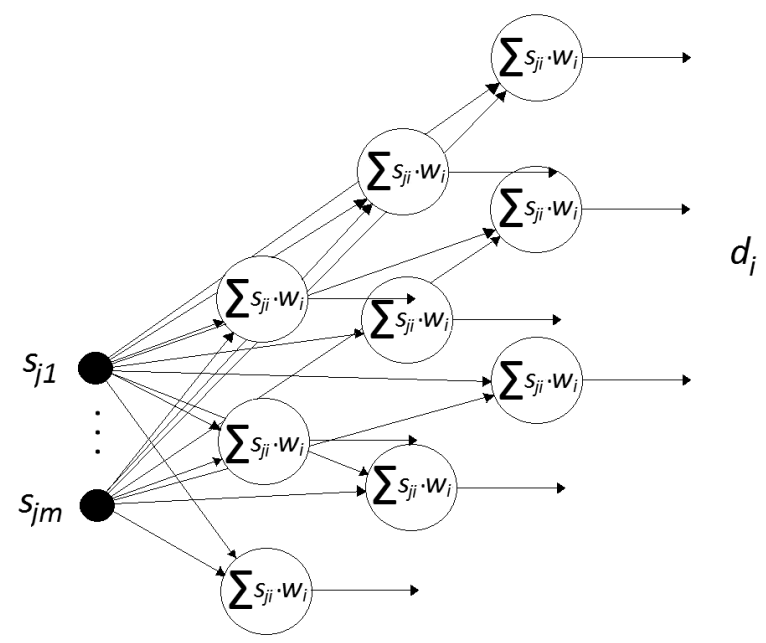

Fig. 11. Structure of the self-organizing map.

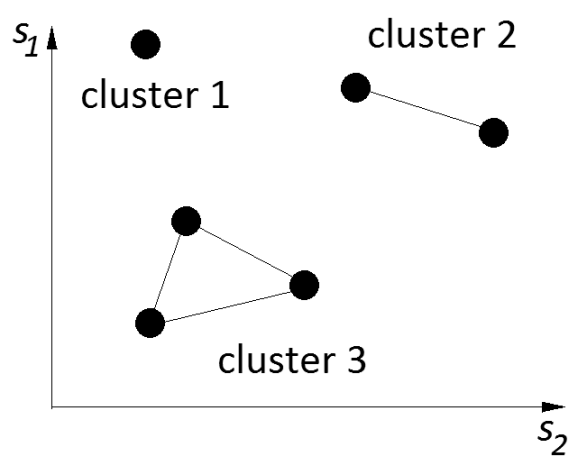

Fig. 12. Structure of graph clustering. 
determine the difficulty of selected SUTs for diagnostics. They process relations between symptoms in data sets. If multiple examples representing different faults are grouped inside a single cluster, they will be difficult to distinguish by the diagnostic method. In this it is possible to determine if the fault detection approaches applied give poor results because of their nature, or because of the difficulty of the data set. In this way, different algorithms can be compared. Alternatively, the method was used to find ambiguity groups in the analog system, represented by clusters containing examples representing different fault categories (Bilski, 2013).

\section{Examples of applications}

Applications to selected systems are presented here. Examples check efficiency and the difficulty of tuning the methods applied. The electronic filter was analyzed using the rough set approach. Problems to solve, apart from the simulations and interpreting the results, included the optimization of RS parameters.

To measure the quality of the fault detection system, (10) was used. As presented by Bilski and Wojciechowski (2011), discretization is of critical importance for diagnostic efficiency. The first experiment was conducted for compact data sets, containing 70 examples ( 7 for each element). As proven in our previous research, the best diagnostic outcomes are for EFB discretization combined with genetic reduction. The optimization of the number of intervals in EFB is more important than proper selection of reduction method parameters. Other discretizations lead to worse results-see Table 1, where the percentages of diagnostic successes are presented.

Genetic reduction requires setting values of the parameters (mutation and crossover probability, the size of the population). Their proper adjustment in many cases increases diagnostic efficiency (Fig. 13). The population size is the most influential parameter. Johnson and Holte's methods give inferior results, although the first algorithm is sometimes comparable to the genetic approach (for EFB discretization). The Holte algorithm produces multiple simple rules and works very fast. Unfortunately, its efficiency is too low to be applicable in practice.

Introduction of larger data sets allowed increasing diagnostic accuracy of the optimal combination of

Table 1. Diagnostic quality for the discretization/reduction combination of the 5th order filter (small data sets)

\begin{tabular}{|c|c|c|c|c|}
\hline Discr. & Genetic & Johnson & Gen. OR & John. OR \\
\hline \hline Boolean & 32.8 & 32.8 & 50.0 & 48.5 \\
\hline Naive & 37.1 & 28.6 & 35.7 & 22.8 \\
\hline S-naive & 32.8 & 28.6 & 32.8 & 22.8 \\
\hline EFB6 & 64.3 & 40.0 & 75.7 & 44.3 \\
\hline EFB8 & 61.2 & 40.0 & 71.4 & 40.0 \\
\hline
\end{tabular}

discretization/reduction (EFB with a genetic algorithm) methods (Table 2). The difference in quality between two pairs of sets is small, although obtaining larger sets required significantly longer simulations. The best results were obtained for 8 intervals in EFB discretization (Fig. 14).

Boolean discretization is the only method producing a single reduct. No stamps excluded from it are discretized. This suggests the possibility to apply another discretization algorithm for these stamps. As experiments show, such an approach does not lead to an increase in accuracy (especially compared with the standalone EFB reduction). Also, Boolean discretization allows using other reduction algorithms: dynamic and exhaustive. They improve RS performance, leading to better results than the genetic algorithm and the Johnson one. Standalone EFB discretization gives the best results.

The induction motor was diagnosed using SVMs.

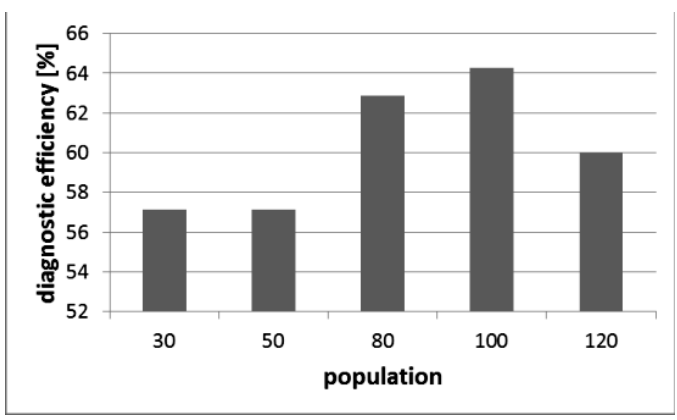

Fig. 13. Influence of the population size in genetic reduction on RS diagnostic efficiency (EFB3 discretization).

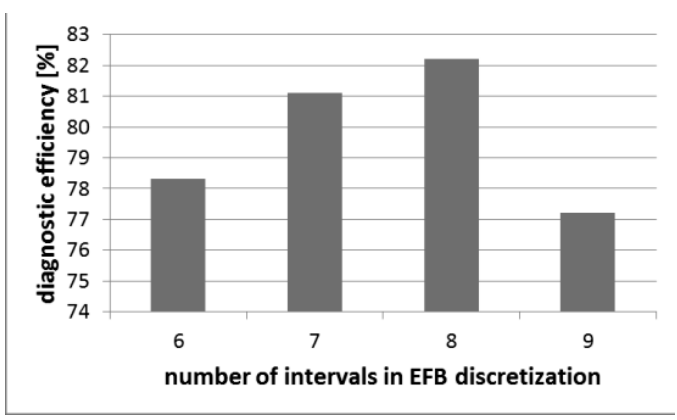

Fig. 14. Influence of the number of intervals in EFB discretization on RS diagnostic efficiency (genetic reduction).

Table 2. Diagnostic accuracy for the discretization/reduction combination of the 5th order filter (large data sets).

\begin{tabular}{|c|c|c|c|}
\hline Discretization & Genetic OR & Johnson OR & Holte \\
\hline \hline Boolean & 63.9 & 60.0 & 5.5 \\
\hline Naive & 47.8 & 15.5 & 22.2 \\
\hline EFB6 & 78.3 & 62.8 & 28.3 \\
\hline EFB8 & 82.2 & 61.1 & 33.3 \\
\hline
\end{tabular}


The aim of the experiment was to approximate values of the SUT parameters, using the error measure (11). To identify the value of each parameter, multiple SVMs had to be used-each for the particular parameter. It was assumed that the regression is the second step in the fault location procedure. First, faulty parameter identification is performed, using a separate classification method. In this way, the efficiency of regression depends on the prior classification procedure.

The size of data sets was changed to determine its influence on the diagnostic quality. Increasing the size of both training and testing sets allowed increasing the accuracy by 80 percent for most parameters. Introduction of tolerances did not degrade regression quality, proving SVM usefulness in uncertainty conditions.

From all available kernels, three were useful, i.e., RBF, ERBF and polynomial. The remaining kernels gave much worse results. The verified kernel values were the width of the radial basis function (for the first two) and the degree of the polynomial (for the third kernel). In the first two cases, acceptable approximation results were obtained for large values of the parameter (such as $10^{6}$ ). The accuracy of SVM regression is shown in Fig. 15.

\section{Conclusions}

Problems and methods presented in the paper demonstrate the importance of diagnostics. Despite widespread presence of digital systems, in some applications their analog counterparts are the only solution. Comparison of modern approaches shows the advantage of heuristic techniques. They are versatile and easy to use. Numerous software tools support their implementation. SUT analysis is then based on the input-output characteristics. After the data set is generated, the presented methods work in the same way, independently of the details of the SUT work mode.

An inconvenience is the need to tune the selected algorithm to a particular case. There is no a-priori

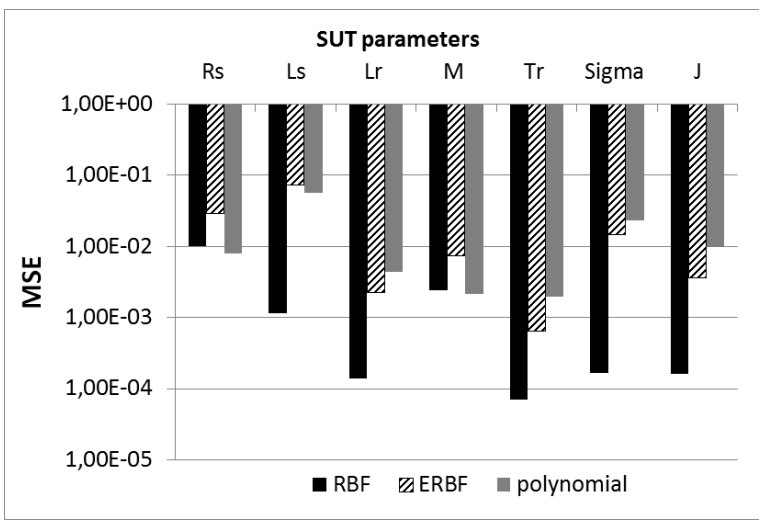

Fig. 15. Regression error for the best SVM kernels. knowledge about the best values of parameters, so additional optimization is performed. Some problems (such as multiple fault identification or analysis of complex systems) still pose a challenge. Therefore, further research on new methods and their combinations is justified. Although computer technologies are advanced enough to apply sophisticated approaches (such as FL or RSs), industrial applications require less hardware with limited computational power. Therefore, it is also important to analyze the complexity of algorithms and their memory requirements. Such problems need to be investigated in the future.

\section{References}

Aminian, F. and Aminian, M. (2001). Fault diagnosis of analog circuits using Bayesian neural networks with wavelet transform as preprocessor, Journal of Electronic Testing 17(1): 29-36.

Anand, G. (2012). Application of artificial neural networks in electrical machines: An overview, World Academy of Science, Engineering and Technology 6(6): 384-385.

Ben Hamida, N. and Kaminska, B. (1993). Multiple fault analog circuit testing by sensitivity analysis, Journal of Electronic Testing 4(4): 331-343.

Betta, G. and Pietrosanto, A. (2000). Instrument fault detection and isolation: State of the art and new research trends, IEEE Transactions on Instrumentation and Measurement 49(1): 100-107.

Bilski, P. and Wojciechowski, J. (2007). Automated diagnostics of analog systems using fuzzy logic approach, IEEE Transactions on Instrumentation Measurement 56(6): 2175-2185.

Bilski, P. and Wojciechowski, J. (2011). Rough-sets-based reduction for analog systems diagnostics, IEEE Transactions on Instrumentation and Measurement 60(3): 880-890.

Bilski, P. (2013). Application of clustering methods for the ambiguity groups detection in the diagnostic of analog systems, Przeglad Elektrotechniczny 89(2a): 276-278.

Bilski, P. and Wojciechowski, J. (2012). Current research trends in diagnostics of analog systems, Proceedings of the International Conference on Signals and Electronic Systems, Wroctaw, Poland, DOI: 10.1109/TIM.2010.2060225.

Browning, T.R. (2001). Applying the design structure matrix to system decomposition and integration problems: A review and new directions, IEEE Transactions on Engineering Management 48(3): 292-306.

Czaja, Z. and Zielonko, R. (2003). Fault diagnosis in electronic circuits based on bilinear transformation in 3D and 4D spaces, IEEE Transactions on Instrumentation and Measurement 52(1): 97-102.

Karki, J. (2002). Active low-pass filter design, Texas Instruments, http://www.ti.com/lit/an/ sloa049b/sloa049b.pdf

Maiden, Y., Jervis Barrie, W., Fouillat, P. and Lesage, S. (1999). Using artificial neural networks or Lagrange interpolation to 
characterize the faults in an analog circuit: An experimental study, IEEE Transactions on Instrumentation and Measurement 48(5): 932-938.

Nowicki, A., Grochowski, M. and Duzinkiewicz, K. (2012). Data-driven models for fault detection using kernel PCA: A water distribution system case study, International Journal of Applied Mathematics and Computer Science 22(4): 939-949, DOI: 10.2478/v10006-012-0070-1.

Patan, K., Witczak, M. and Korbicz, J. (2008). Towards robustness in neural network based fault diagnosis, International Journal of Applied Mathematics and Computer Science 18(4): 443-454, DOI: 10.2478/v10006-008-0039-2.

Pöyhönen, S., Negrea, M., Arkkio, A., Hyötyniemi, H. and Koivo, H. (2002). Fault diagnostics of an electrical machine with multiple support vector classifiers, Proceedings of the 17th IEEE International Symposium on Intelligent Control, Vancouver, British Columbia, Canada, Vol. 1, pp. 373-378.

Rutkowski, J. and Grzechca, D. (2001). Analog fault dictionary-fuzzy set approach, Proceedings of the European Conference on Circuit Theory and Design, Helsinki, Finland, pp. 253-256.

Rutkowski, J. and Zieliński, L. (2003). Using evolutionary techniques for chosen optimization problems related to analog circuits design, Proceedings of the 16th European Conference on Circuits Theory and Design, Cracow, Poland, Vol. 3, pp. III-313-316.

Sałat, R. and Osowski, S. (2011). Support vector machine for soft fault location in electrical circuits, Journal of Intelligent and Fuzzy Systems 22(1): 21-31.

Samanta, B. and Nataraj, C. (2009). Use of particle swarm optimization for machinery fault detection, Engineering Applications of Artificial Intelligence 22(2): 308-316.

Simani, S. (2013). Residual generator fuzzy identification for automotive diesel engine fault diagnosis, International Journal of Applied Mathematics and Computer Science 23(2): 419-438, DOI: 10.2478/amcs-2013-0032.

Smyth, P. (1994). Hidden Markov models for fault detection in dynamic systems, Pattern Recognition 27(1): 149-164.

Starzyk, J.A., Pang, J., Manetti, S., Piccirilli, M.C. and Fedi, G. (2000). Finding ambiguity groups in low testability analog circuits, IEEE Transactions on Circuits and Systems 47(8): $1125-1137$.

Starzyk, J., Liu, D., Liu, Z., Nelson, D. and Rutkowski, J. (2004). Entropy-based optimum test points selection for analog fault dictionary techniques, IEEE Transactions on Instrumentation and Measurement 53(3): 754-761.

Svensson, M., Byttner, S. and Rognvaldsson, T. (2008). Self-organizing maps for automatic fault detection in a vehicle cooling system, Proceedings of the 4th International IEEE Conference on Intelligent Systems, Varna, Bulgaria, Vol. 3, pp. 8-12.

Tadeusiewicz, M. and Hałgas, S. (2007). Finding operating points of the diode-transistor circuits via homotopy approach, Przeglad Elektrotechniczny 83(2): 69-72.
Tadeusiewicz, M., Hałgas, S. and Korzybski, M. (2011). Multiple catastrophic fault diagnosis of analog circuits considering the component tolerances, International Journal on Circuits Theory and Applications 40(10): 1041-1052, DOI: $10.1002 /$ cta.770.

Tudoroiu, N. and Zaheeruddin, M. (2005). Fault detection and diagnosis of valve actuators in HVAC systems, Proceedings of the 2005 IEEE Conference on Control Applications, Toronto, Canada, pp. 1281-1286.

Wang, K., Chiasson, J., Bodson, M. and Tolbert, L.M. (2007) An online rotor time constant estimator for the induction machine, IEEE Transactions on Control Systems Technology 15(2): 339-348.

Wu, S. and Chow, T.W.S. (2004). Induction machine fault detection using SOM-based RBF neural networks, IEEE Transactions on Industrial Electronics 51(1): 183-194.

Xue, H. and Jiang, J.G. (2006). Fault detection and accommodation for nonlinear systems using fuzzy neural networks, Proceedings of the 5th International Power Electronics and Motion Control Conference, Shanghai, China, pp. 1-5, DOI: 10.1109/IPEMC.2006.4778342.

Zhu, L., Zhu, Y., Mao, H. and Gu, M. (2009). A new method for sparse signal denoising based on compressed sensing, 2nd International Symposium on Knowledge Acquisition and Modeling, Wuhan, China, pp. 35-38.

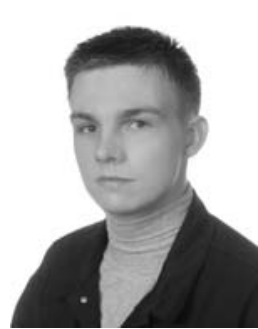

Piotr Bilski obtained his M.Sc. in 2001 and his Ph.D. in 2006 (both with honors). Currently he is an assistant professor at the Institute of Radioelectronics, Warsaw University of Technology, and the Department of Applied Informatics, Warsaw University of Life Sciences. His main scientific interests are diagnostics of analog systems, design and analysis of virtual instrumentation, as well as application of artificial intelligental sciences ce and machine learning methods to environmen-

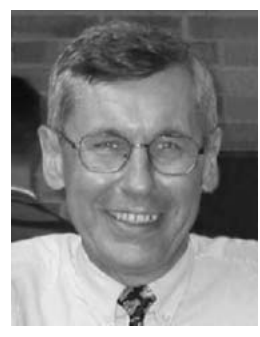

Jacek Wojciechowski received his M.Sc., Ph.D. (with honors) and D.Sc. degrees from the Faculty of Electronics, Warsaw University of Technology, Poland, in 1966, 1976, and 1989, respectively. $\mathrm{He}$ also received an M.Sc. degree in mathematics from the University of Warsaw in 1975. Currently he is a full professor at the Institute of Radioelectronics, Warsaw University of Technology. He is the author or a co-author of over 100 technical publications and six books. He currently serves as an associate editor for the Journal of the Franklin Institute. His research interests cover systems engineering (simulation and design for quality, sensitivity analysis, fault location in analog and switched systems), information and communication systems, networks and graphs, and heuristic methods (e.g., evolutionary algorithms and fuzzy logic).

Received: 21 January 2013

Revised: 28 February 2013 